\title{
Uso da subirrigação para imposição de estresse hídrico em sistema semi-contínuo para medição de $\mathrm{CO}_{2}{ }^{(1)}$
}

\author{
RHUANITO SORANZ FERRAREZ|(2)*, MARC W. VAN IERSEL ${ }^{(2)}$ e ROBERTO TESTEZLAF ${ }^{(3)}$
}

\begin{abstract}
RESUMO
Os objetivos deste trabalho foram avaliar os efeitos de valores distintos de umidade do substrato para acionamento da subirrigação sobre a fotossíntese e o crescimento de plantas de sálvia e avaliar a viabilidade do uso da subirrigação em pesquisas relacionadas à imposição de estresse hídrico nessa cultura. Os tratamentos foram dois conteúdos volumétricos de água (CVA, 0,2 e 0,4 $\mathrm{m}^{3} \mathrm{~m}^{-3}$ ) para acionamento da subirrigação, com quatro repetições. Cada repetição era composta por 10 plantas. Utilizou-se um sistema automatizado semi-contínuo para medição de $\mathrm{CO}_{2}$ com múltiplas câmaras de cultivo, com sensores capacitivos para o monitoramento contínuo da umidade no substrato. A subirrigação com solução nutritiva foi realizada manualmente quando o CVA atingia valores abaixo dos pré-estabelecidos. Os valores médios de fotossíntese líquida, ganho de carbono por dia e eficiência do uso de carbono em ambos os tratamentos reduziram ao longo do tempo, de 2 para $1,1 \mu \mathrm{mol} \mathrm{s}{ }^{-1}, 2,2$ para $1 \mu \mathrm{mol} \mathrm{d}^{-1}$ e de 0,7 para $0,45 \mathrm{~mol} \mathrm{~mol}^{-1}$, respectivamente. A massa seca total da parte aérea $(p=0,0129)$, a altura da parte aérea na ponta da flor mais alta $(p<0,0001)$ e a área foliar total $(p=0,0007)$ foram estatisticamente superiores no tratamento $0,4 \mathrm{~m}^{3} \mathrm{~m}^{-3}$. O uso da subirrigação nesse experimento não permitiu a imposição do estresse hídrico, pois houve variação excessiva no valor do CVA após as irrigações, em ambos os tratamentos. O tratamento com valor mais elevado de umidade ocasionou resposta positiva no crescimento das plantas de sálvia cultivadas por subirrigação.

Palavras-chave: Salvia splendens 'Vista Rose’, estresse hídrico, fotossíntese, sensores de umidade.
\end{abstract}

\section{ABSTRACT}

Use of subirrigation for water stress imposition in a semi-continuous $\mathrm{CO}_{2}$-exchange system

The objectives of this work were to evaluate the effects of distinct moisture contents to trigger subirrigation on salvia photosynthesis and plant growth, and to verify the feasibility of subirrigation use in water stress imposition research in this crop. We evaluated two substrate volumetric water contents (VWC) as treatments $\left(0.2\right.$ and $\left.0.4 \mathrm{~m}^{3} \mathrm{~m}^{-3}\right)$ to trigger subirrigation, with 4 replications. Each replication was composed of 10 plants. An automated semi-continuous multi-chamber $\mathrm{crop}^{\mathrm{CO}_{2}-}$ exchange system was used, with capacitance soil moisture sensors for continuous moisture monitoring. Manual subirrigation with nutrient solution was performed when VWC dropped below the thresholds. In both treatments, the values of net photosynthesis, daily carbon gain and carbon use efficiency reduced over time, from 2 to $1.1 \mu \mathrm{mol} \mathrm{s} \mathrm{s}^{-1}$ from 2.2 to $1 \mu \mathrm{mol} \mathrm{d}^{-1}$ from 0.7 to $0.45 \mathrm{~mol} \mathrm{~mol}^{-1}$, respectively, in both soil moisture treatments. Total shoot dry mass $(\mathrm{p}=0.0129)$, shoot height in the tip of the highest flower $(\mathrm{p}<0.0001)$ and total leaf area $(\mathrm{p}=0.0007)$ were statistically higher at $0.4 \mathrm{~m}^{3} \mathrm{~m}^{-3}$ treatment. The subirrigation system was not efficient to impose water stress, due to excessive variation on VWC values after each irrigation event in both treatments. Higher soil moisture promoted positive plant growth responses in salvia cultivated by subirrigation. Keywords: Salvia splendens 'Vista Rose', drought stress, photosynthesis, soil moisture sensors.

\section{INTRODUÇÃO}

A compreensão do comportamento das plantas sob condições de déficit hídrico se faz necessária porque as mudanças climáticas podem reduzir a disponibilidade de água para algumas regiões do planeta (KIM e VAN IERSEL, 2011). O déficit hídrico limita a produtividade das culturas agrícolas (CHAVES et al., 2009). Mecanismos morfofisiológicos como fotossíntese, senescência e abscisão foliar são severamente afetados no caso de ocorrência repentina de falta de água, enquanto que sua ocorrência gradual e/ou no início do ciclo promove a rápida adaptação das plantas (SANTOS e CARLESSO, 1998). Por outro lado, as plantas cultivadas em condições adequadas de umidade são menos resistentes ao déficit hídrico, o que influencia diretamente a taxa fotossintética (MARQUES et al., 2011).

A fotossíntese é um processo primário na produção de biomassa vegetal, produzindo energia na forma de ATP para crescimento e desenvolvimento das plantas. Este processo tem sido amplamente estudado nas últimas décadas, principalmente por meio do uso de equipamentos que realizam sua medida em nível foliar (VAN IERSEL e BUGBEE, 2000). No entanto, alguns autores (ZELITCH, 1982, ANDRIOLO et al., 1996 e VAN IERSEL e BUGBEE, 2000) indicaram que os métodos de medição baseados na planta inteira são mais eficientes, principalmente porque permitem a correlação dos dados

\footnotetext{
(1) Trabalho recebido para publicação em 23/10/2014 e aprovado em 26/03/2015

(2) University of Georgia (UGA), Department of Horticulture, Athens/GA, Department of Horticulture, Athens-GA, United States of America. *Autor

correspondente: rhuanito@terra.com.br

(3) Universidade Estadual de Campinas (UNICAMP), Faculdade de Engenharia Agrícola (FEAGRI), Campinas/SP, Campinas-SP, Brasil.
} 
obtidos com a produção de massa seca e produtividade e por superar o problema de selecionar a folha adequada para realizar a medição. Além disso, permitem medições não destrutivas do crescimento vegetal e também o controle das condições ambientais $\left(\mathrm{CO}_{2}\right.$, temperatura, luz e umidade relativa), do substrato (umidade e evaporação) e da planta (fotossíntese e transpiração), eliminando flutuações naturais e imprevisíveis que dificultam a interpretação dos resultados. Medições contínuas da taxa de trocas gasosas de carbono por períodos prolongados também tornam possível a determinação do momento de ocorrência do efeito dos tratamentos, o que é, em geral, difícil de detectar por técnicas tradicionais como determinação da massa seca acumulada ou medidas pontuais de fotossíntese nas folhas (VAN IERSEL e KANG, 2002).

A subirrigação, técnica difundida no exterior e pouco utilizada em sistemas de cultivo em ambiente protegido no Brasil, utiliza o princípio de capilaridade para realizar o umedecimento dos substratos (FERRAREZI et al., 2014). Esse sistema de irrigação aplica água na base dos recipientes de cultivo, possibilitando nutrição adequada das plantas, reutilização de solução nutritiva e redução do descarte de soluções ricas em nutrientes no solo das estufas, o que elimina a contaminação de lençóis freáticos. A subirrigação também é uma alternativa para aumentar a uniformidade e precocidade da produção (FERRAREZI et al., 2015).

Há poucos trabalhos que avaliam diferentes umidades do substrato para acionamento da subirrigação. Ferrarezi et al. (2015) cultivaram hibiscos (Hibiscus acetosella 'Panama Red') e Ferrarezi et al. (2014) produziram sálvia (Salvia splendens 'Vista Red') em um sistema automatizado baseado em valores mínimos de umidade do substrato para acionamento da subirrigação. Esses autores observaram maior variação da umidade quando o valor usado para acionamento foi de $0,1 \mathrm{~m}^{3} \mathrm{~m}^{-3}$ comparado com valores maiores que $0,42 \mathrm{~m}^{3} \mathrm{~m}^{-3}$. Embora o efeito positivo da subirrigação no crescimento vegetal seja conhecido, a resposta das plantas na realização de fotossíntese ainda foi pouco estudada. A praticidade de aplicação de água usando esse método poderia justificar seu emprego na imposição de déficit hídrico em estudos relacionados com a fotossíntese em câmaras fechadas para determinação do $\mathrm{CO}_{2}$ em planta inteira.

Os objetivos deste trabalho foram avaliar os efeitos de valores distintos de umidade do substrato para acionamento da subirrigação sobre a fotossíntese e o crescimento de plantas de sálvia cultivadas em sistema automatizado semi-contínuo para medição de $\mathrm{CO}_{2}$ em planta inteira e avaliar a viabilidade do uso da subirrigação em pesquisas relacionadas à imposição de estresse hídrico nessa cultura.

\section{MATERIAL E MÉTODOS}

O experimento foi realizado no Departamento de Horticultura da University of Georgia (UGA), em Athens/ GA, Estados Unidos da América (EUA).

Mudas de Salvia splendens Sell ex Roem. \& Schult. 'Vista Rose' com 30 dias após semeadura, fornecidas por um viveiro comercial (Knox Nursery, Winter Garden/FL, EUA), foram transplantadas em 23/06/2011 (denominado dia após o transplantio ou DAT 1). Utilizaram-se vasos quadrados com dimensões de 10 x 10 × $9 \mathrm{~cm}$ (comprimento $\mathrm{x}$ largura $\mathrm{x}$ altura), preenchidos com substrato Fafard $1 \mathrm{P}$ (Conrad Fafard, Agawam/MA, EUA) com 80\% de turfa e $20 \%$ de perlita. O experimento foi conduzido por 22 dias (de 18/07 - DAT 26 a 08/08/2011 - DAT 48). O valor médio da massa seca total da parte aérea no início da aplicação dos dois tratamentos foi de 1,635 g planta $^{-1}$.

As plantas foram acondicionadas em duas câmaras de crescimento E-15, PGR15 (Conviron Winnipeg/Manitoba, Canadá), contendo quatro câmaras de acrílico de 47 x 36 x $61 \mathrm{~cm}$ cada, utilizadas para medir as trocas de $\mathrm{CO}_{2}$ realizadas pela planta inteira. Sistemas automatizados coletavam e armazenavam a cada $10 \mathrm{~min}$ os valores de fotossíntese de maneira semi-contínua nas câmaras. As medições levavam 1 min por câmara, sendo os primeiros $30 \mathrm{~s}$ empregados nas leituras, e os outros $30 \mathrm{~s}$, utilizados para expulsar o ar da medida anterior da tubulação. Detalhes construtivos do sistema podem ser obtidos em Kim et al. (2011) e van Iersel e Bugbee (2000). As câmaras de crescimento possuíam lâmpadas fluorescentes ajustadas para fornecer fluxo de fótons fotossinteticamente ativos (photosynthetic photon flux, PPF) na altura do dossel de $350 \mu \mathrm{mol} \mathrm{m} \mathrm{m}^{-2} \mathrm{~s}^{-1}$, com fotoperíodo de $12 \mathrm{~h}$.

Cada câmara de acrílico foi equipada com um sensor de umidade do substrato EC-5, instalado em um dos vasos, conectado a um data logger EM-50 (ambos da Decagon Devices, Pullman/WA, EUA) para monitoramento da umidade e determinação do momento adequado para irrigação. A temperatura foi mantida a $25 \pm 1^{\circ} \mathrm{C}$ dentro das câmaras de acrílico por meio de resistências elétricas, monitorada por um termopar tipo $\mathrm{T}$ conectado a um multiplexador AM25T (Campbell Scientific, Logan/UT, EUA). A umidade relativa foi mantida de 65 a $85 \%$ durante o dia e de 80 a $100 \%$ durante a noite, sendo monitorada por sensores de umidade HTO-45R (Rotronic, Huntington/NY, EUA). $\mathrm{O}$ ar no interior de cada câmara de troca gasosa foi homogeneizado por um ventilador.

Os tratamentos testados foram dois conteúdos volumétricos de água (CVA) do substrato $\left(0,2\right.$ e $\left.0,4 \mathrm{~m}^{3} \mathrm{~m}^{-3}\right)$ para acionamento da subirrigação, não havendo a pretensão de manter a umidade do substrato nos valores estabelecidos como tratamentos, pois sistemas de irrigação baseados no gotejamento têm sido usados com sucesso para essa finalidade (CHAPPELL et al., 2013 e LEA-COX et al., 2013). Os tratamentos foram arranjados em delineamento de blocos ao acaso, com quatro repetições, totalizando oito unidades experimentais. Cada unidade experimental constituiu-se de 10 plantas, dispostas em uma bandeja plástica com $45 \times 30 \times 5 \mathrm{~cm}$.

Medidas de umidade do substrato foram realizadas pelos sensores capacitivos a cada $20 \mathrm{~min}$, e os dados coletados e armazenados a cada $2 \mathrm{~h}$. Toda vez que o CVA do substrato foi inferior aos limites pré-estabelecidos pelos tratamentos $\left(0,2\right.$ e $\left.0,4 \mathrm{~m}^{3} \mathrm{~m}^{-3}\right)$, aplicou-se manualmente aproximadamente $80 \mathrm{~mL}$ de água por vaso via subirrigação até saturação do substrato. A solução não utilizada pelas 
plantas era mantida na bandeja plástica situada abaixo dos vasos até evaporação completa. Utilizou-se uma solução nutritiva para as subirrigações preparada com o fertilizante solúvel 15-5-15 Cal-Mag Special (Scotts Co., Marysville/ $\mathrm{OH}, \mathrm{EUA})$, com condutividade elétrica (CE) de $0,59 \mathrm{mS}$ $\mathrm{cm}^{-1}$ e a seguinte composição de nutrientes $\left(\mathrm{mg} \mathrm{L}^{-1}\right): \mathrm{N}$ total (100) $\left[8 \% \mathrm{NH}_{4}^{+}, 78,3 \% \mathrm{NO}_{3}^{-}\right.$e $13,7 \%$ uréia], $\mathrm{P}(33,3)$, $\mathrm{K}(100,0), \mathrm{Ca}(33,3), \mathrm{Mg}(13,3), \mathrm{B}(0,12), \mathrm{Cu}(0,12), \mathrm{Fe}$ $(0,50), \operatorname{Mn}(0,25)$, Mo $(0,05)$ e $\mathrm{Zn}(0,25)$.

A fotossíntese bruta (fotossíntese na presença de luz + respiração no escuro) (Eq. 1) foi determinada ao longo do período experimental por um analisador infravermelho de trocas gasosas $\mathrm{CO}_{2}$ em modo diferencial LI-6262 (Li-COR Biosciences, Lincoln/NE, EUA). A partir da fotossíntese bruta se determinaram ganho diário de carbono (DCG, a quantidade líquida de carbono fixada durante um período de 24 h) (Eq. 2) e eficiência do uso de carbono (CUE) (Eq. 3) (VAN IERSEL e KANG, 2002). De acordo com van Iersel e Kang (2002), o produto $F_{B} x$ tempo de luz é a quantidade estimada de carbono fixada na fotossíntese bruta durante um período de $24 \mathrm{~h}$.

$\mathrm{F}_{\mathrm{B}}=\mathrm{F}_{\mathrm{L}}+\mathrm{R}_{\mathrm{E}}$

$\mathrm{DCG}=\left(\mathrm{F}_{\mathrm{L}} \mathrm{x}\right.$ tempo de luz $)-\left(\mathrm{R}_{\mathrm{E}} \mathrm{x}\right.$ tempo de escuro $)($ Eq. 2$)$

$\mathrm{CUE}=\mathrm{DCG} /\left(\mathrm{F}_{\mathrm{B}} \mathrm{x}\right.$ tempo de luz $)$

Em que: $\mathrm{F}_{\mathrm{B}}=$ fotossíntese bruta, $\mu \mathrm{mol} \mathrm{s} \mathrm{s}^{-1} ; \mathrm{F}_{\mathrm{L}}=$ fotossíntese líquida na presença de luz, $\mu \mathrm{mol} \mathrm{s}^{-1}$; e $\mathrm{R}_{\mathrm{E}}=$ respiração no escuro, $\mu \mathrm{mol} \mathrm{s}{ }^{-1}$.

Ao final do experimento foram determinadas a massa seca das folhas, dos caules + flores e total da parte aérea, a altura da parte aérea na ponta da flor mais alta e na axila foliar mais alta, a área foliar total usando o integrador LI3100 (Li-COR Biosciences, Lincoln/NE, EUA) e o número de ramos.
Os resultados obtidos foram submetidos à análise de variância (ANOVA) e teste de comparação de médias de Tukey, usando o software estatístico SAS 9.2 (SAS Institute, Cary/NC, EUA).

\section{RESULTADOS E DISCUSSÃO}

O processo de evapotranspiração da cultura provocou redução da umidade do substrato até o nível estabelecido para acionamento manual da subirrigação em ambos os tratamentos (Figura 1). Algumas repetições atingiram valores de CVA ligeiramente abaixo do estabelecido para o tratamento, pois utilizou-se irrigação não automatizada. Após cada evento de irrigação, foi possível verificar que houve elevação nos valores de umidade do substrato, com os valores de CVA apresentando uma amplitude de variação de 0,1873 a 0,6545 $\mathrm{m}^{3} \mathrm{~m}^{-3}$ (Figura 1A) e de 0,3852 a $0,6834 \mathrm{~m}^{3} \mathrm{~m}^{-3}$ (Figura 1B) para os tratamentos 0,2 e $0,4 \mathrm{~m}^{3} \mathrm{~m}^{-3}$, respectivamente.

Esses resultados foram similares aos encontrados por Ferrarezi et al. (2015) trabalhando com hibiscos em mesas de subirrigação tipo ebb-and-flow. De acordo com Kim et al. (2011) a umidade utilizada para produção das principais ornamentais de interesse econômico está na faixa de 0,4 $\mathrm{m}^{3} \mathrm{~m}^{-3}$. Esperava-se, portanto, que o tratamento com umidade de $0,2 \mathrm{~m}^{3} \mathrm{~m}^{-3}$ pudesse causar estresse hídrico, porém o aumento da umidade do substrato forneceu água em quantidade suficiente para o desenvolvimento da cultura. A subirrigação não se mostrou adequada para manutenção de valores estáveis de CVA, conforme ocorreu em um prototipo indicado por Snow e Tangey (1985). Essa ausência de resposta pode ter sido decorrente da diferença de equipamentos utilizados nos estudos, pois quando se comparam equipamentos modernos os resultados são similares. Ferrarezi et al. (2015) e Ferrarezi et al. (2014) também encontraram grandes variações no CVA logo após a subirrigação em mesas tipo ebb-and-flow no cultivo de hibiscos e sálvia.

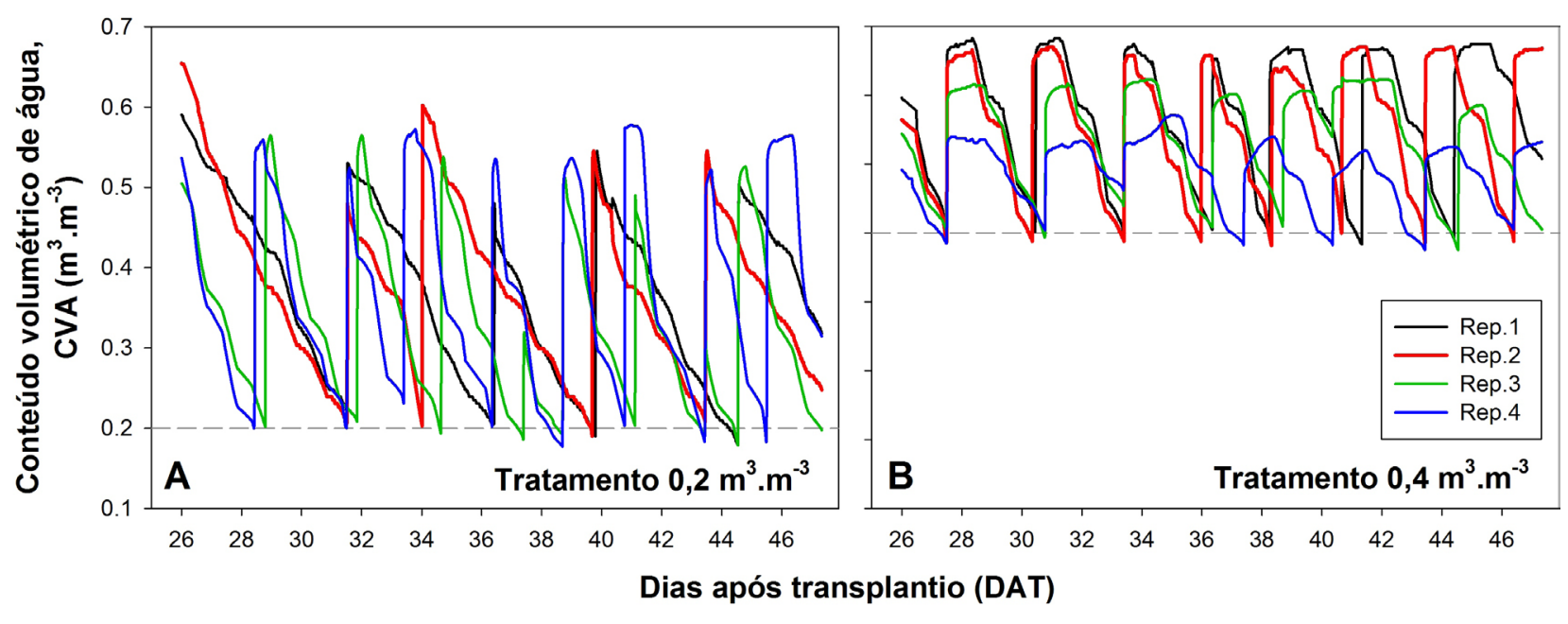

Figura 1. Conteúdo volumétrico de água (CVA) do substrato ao longo do período experimental nos valores de umidade para acionamento da subirrigação 0,2 (A) e $0,4 \mathrm{~m}^{3} \mathrm{~m}^{-3}(\mathrm{~B})$. A linha pontilhada indica o limite de umidade para aplicação manual da subirrigação.

Figure 1. Volumetric water content (VWC) throughout the experiment in two substrate moisture values to trigger subirrigation: $0.2(A)$ and $0.4 \mathrm{~m}^{3} \mathrm{~m}^{-3}(B)$. The dotted line indicates the threshold for manual application of subirrigation. 
Valores acima de zero indicam realização de fotossíntese pelas plantas, enquanto valores abaixo de zero evidenciam o processo respiratório (Figuras $2 \mathrm{~A}$ e 2B). Observaram-se valores elevados da fotossíntese no início do experimento variando de 1,2 a $2,2 \mu \mathrm{mol}$ $\mathrm{s}^{-1}$, com redução gradual ao longo do tempo de 0,6 a $1,2 \mu \mathrm{mol} \mathrm{s} \mathrm{s}^{-1}$ para os dois tratamentos (Figuras $2 \mathrm{~A} \mathrm{e}$ 2B). Os valores médios de fotossíntese em ambos os tratamentos foram reduzidos em aproximadamente 50\% ao longo do tempo, passando respectivamente de 2,0 para $1,1 \mu \mathrm{mol} \mathrm{s} \mathrm{s}^{-1}$. Este valor ficou abaixo do esperado até o término do experimento. Resultados similares foram encontrados no trabalho de Kang e van Iersel (2004), que testaram valores crescentes de $\mathrm{CE}$ e pH no desenvolvimento de sálvia e observaram redução na fotossíntese e transpiração. No entanto, não houve diferença significativa para essa variável entre os dois tratamentos testados nesse experimento $(\mathrm{p}>0,05)$. Observou-se que os picos de elevação na fotossíntese estão relacionados com o aumento do CVA provocados pelas irrigações (Figura 1), o que também foi encontrado por Kim et al. (2011).

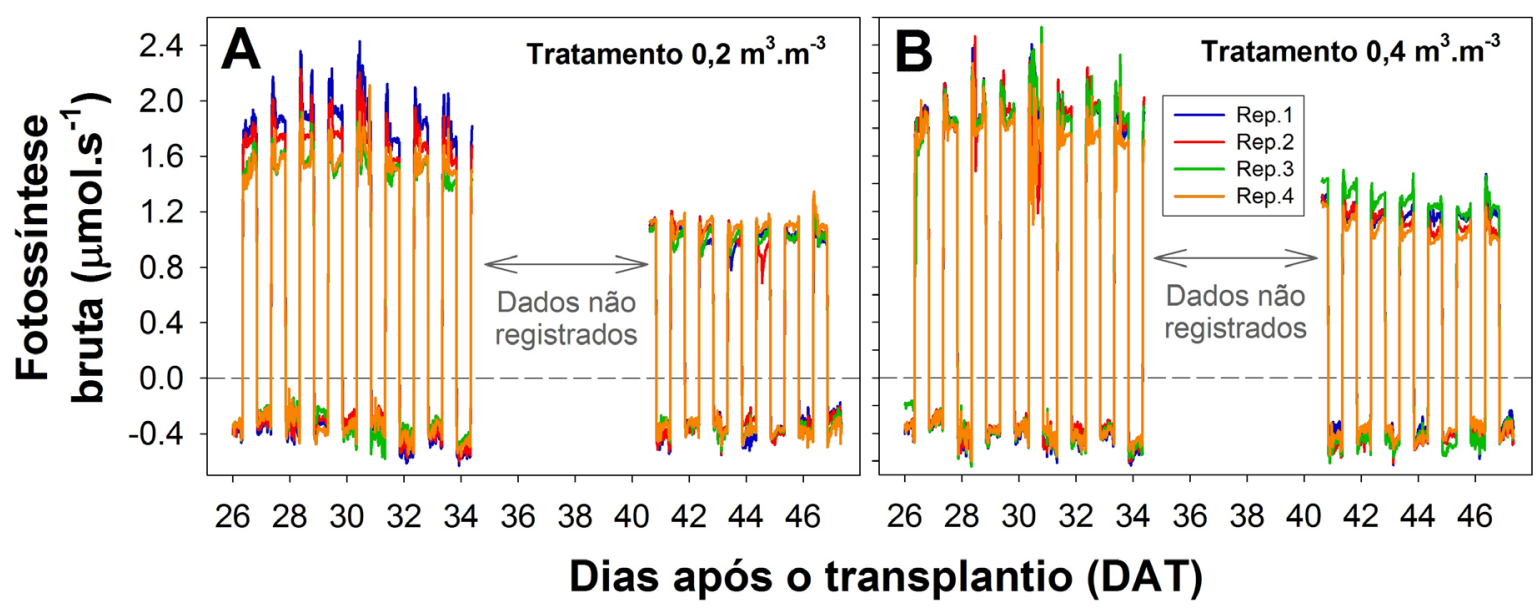

Figura 2. Fotossíntese bruta de Salvia splendens Sell ex Roem. \& Schult. 'Vista Rose' ao longo do período experimental nos valores de umidade para acionamento da subirrigação 0,2 (A) e 0,4 $\mathrm{m}^{3} \mathrm{~m}^{-3}$ (B). Observação: os dados não foram registrados do DAT 34 até o DAT 40 por falha no sistema de aquisição, ocasionando a interrupção no armazenamento das medições de fotossíntese.

Figure 2. Gross photosynthesis throughout the experiment in two substrate moisture values to trigger subirrigation: 0.2 (A) and $0.4 \mathrm{~m}^{3} \mathrm{~m}^{-3}$ (B). Note: Data were not recorded from DAT 34 to 40 due to a fail in the data acquisition system, causing disruption in the storage of photosynthesis measurements.

O ganho diário de carbono (DCG), que é uma medida da taxa de crescimento das plantas, pode ser visualizado na Figura 3B. O tratamento $0,4 \mathrm{~m}^{3} \mathrm{~m}^{-3}$ apresentou valores significativamente superiores ao tratamento $0,2 \mathrm{~m}^{3} \mathrm{~m}^{-3}$ no início do experimento $(\mathrm{p}<0,05)$. Os valores do $\mathrm{DCG}$ reduziram em média de 2,2 para $1,0 \mu \mathrm{mol} \mathrm{d}^{-1}$ ao longo do tempo em ambos os tratamentos (Figura 3B), o que corresponde a $55 \%$ do valor inicial. Essa redução foi causada pela diminuição na fotossíntese, que reduziu, consequentemente, o fornecimento de carbono para o ganho de massa das plantas. Esses resultados são contrários aos encontrados por Kim et al. (2011), Nemali e van Iersel (2004) e van Iersel e Kang (2002), que mostraram que à medida que a planta cresce e se desenvolve estimulada por água, luminosidade ou nutrientes, o valor de DCG aumenta.

A variação da eficiência do uso de carbono (CUE) pelas plantas ao longo do ciclo para o tratamento $0,2 \mathrm{~m}^{3}$ $\mathrm{m}^{-3}$ apresentou valores superiores ao longo da maioria do período experimental, com significância somente no DAT $28(\mathrm{p}<0,05)$ (Figura 3C). Aos 26, 32 e 33 DAT houve efeito significativo somente para o tratamento $0,4 \mathrm{~m}^{3} \mathrm{~m}^{-3}$. Foi possível verificar que os valores médios da CUE reduziram de 0,7 para $0,45 \mathrm{~mol} \mathrm{~mol}^{-1}$ para ambos os tratamentos ao longo do tempo, indicando menor eficiência das plantas em converter o carbono assimilado pela fotossíntese em ganho de massa seca (Figura 3C). Esse resultado também é contrário ao encontrado por van Iersel e Bugbee (2000), van Iersel e Kang (2002) e Nemali e van Iersel (2004), onde houve aumento nesse valor.

Uma possível explicação para esses resultados decrescentes na fotossíntese, DCG e CUE pode ter sido incremento da umidade do substrato logo após o acionamento da subirrigação (Figura 1). A lâmina de água temporária que ficou na parte inferior dos vasos proporcionou o aumento da umidade do substrato. Isso não se constitui numa desvantagem da subirrigação; somente inviabiliza a aplicação de valores controlados de umidade em experimentos científicos. O CVA de $0,2 \mathrm{~m}^{3} \mathrm{~m}^{-3}$ proporcionou baixa umidade do substrato que no entanto, foi seguido de valores bem acima de $0,4 \mathrm{~m}^{3} \mathrm{~m}^{-3}$ após as irrigações. Essa variação pode ter resultado em decréscimo na oxigenação das raízes causado pela saturação e possível ocorrência de hipoxia, efeito prejudicial para a fotossíntese (GARCÍA-SÁNCHEZ et al., 2007) e, consequentemente, 


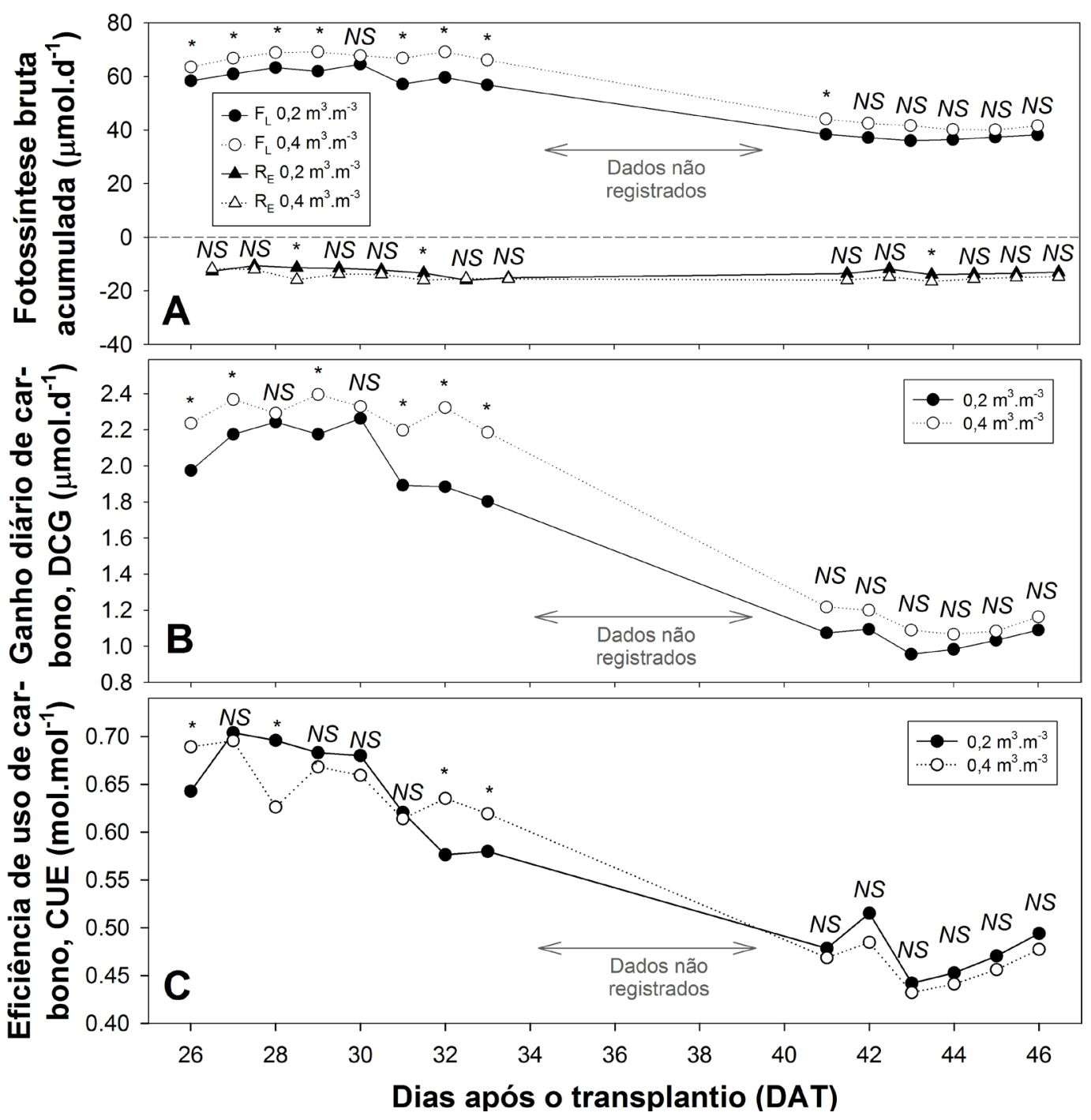

Figura 3. Fotossíntese bruta acumulada (A), ganho diário de carbono, DCG (B) e eficiência do uso de carbono, CUE (C) de Salvia splendens Sell ex Roem. \& Schult. 'Vista Rose' ao longo do período experimental nos valores de umidade para acionamento da subirrigação 0,2 e $0,4 \mathrm{~m}^{3} \mathrm{~m}^{-3}$. Observação: os dados não foram registrados do DAT 34 até o DAT 40 por falha no sistema de aquisição, ocasionando a interrupção no armazenamento das medições de fotossíntese e, consequentemente, do DCG e CUE. Onde: NS = não significativo, $\mathrm{e}^{*}=$ significativo pelo teste de comparação de médias de Tukey a $5 \%$ de probabilidade.

Figure 3. Accumulated gross photosynthesis (A), daily carbon gain, DCG (B) and carbon use efficiency, CUE (C) throughout the experiment in two substrate moisture values to trigger subirrigation: 0.2 and $0.4 \mathrm{~m}^{3} \mathrm{~m}^{-3}$. Note: Data were not recorded from DAT 34 to 40 due to a fail in the data acquisition system, causing disruption in the storage of photosynthesis and, consequently, DCG and CUE measurements. Where: NS = not significant, and $*=$ Tukey mean comparison test was significant at 5\% probability.

para o desenvolvimento vegetal (PISICCHIO et al., $2010 \mathrm{e}$ VAN IERSEL et al., 2010). Esses resultados também estão de acordo com Snow e Tingey (1985), que avaliaram um sistema para imposição de estresse hídrico em plantas e encontraram que a severidade e o padrão de desenvolvimento do estresse estavam relacionados com a altura da coluna de água e com a condutividade hidráulica do substrato. Como plantas cultivadas em condições adequadas de umidade são menos resistentes ao déficit hídrico, com influência direta na fotossíntese (SANTOS e CARLESSO, 1998), os resultados desse experimento indicam que o menor CVA do tratamento $0,2 \mathrm{~m}^{3} \mathrm{~m}^{-3}$ reduziu a fotossíntese ao longo do tempo. Porém, efeito similar ocorreu com o tratamento $0,4 \mathrm{~m}^{3} \mathrm{~m}^{-3}$, indicando que a variação de umidade nos dois substratos após as irrigações foi prejudicial para o desenvolvimento das plantas. Deve-se ainda citar que houve um problema não detectado no arquivo de coleta de dados, que ocasionou a interrupção no armazenamento das medições de fotossíntese do DAT 34 até o DAT 40, porém sem relação com o sistema semi-contínuo de medição de $\mathrm{CO}_{2}$, que funcionou adequadamente ao longo de todo o período experimental. 
Não houve efeito significativo dos tratamentos na massa seca das folhas $(p>0,05)$, porém houve efeito significativo para a massa seca dos caules + flores $(p=0,0123)$ e massa seca total da parte aérea $(\mathrm{p}=0,0129)$, com resultados respectivamente $18 \%$ e $10 \%$ superiores no tratamento $0,4 \mathrm{~m}^{3}$ $\mathrm{m}^{-3}$, com maior umidade para acionamento da subirrigação (Tabela 1). A subirrigação possui a característica de elevar a CE nas camadas superiores do substrato e reduzir o crescimento vegetal (KANG e VAN IERSEL, 2004). Porém ao longo desse experimento não houve alteração do valor inicial da $\mathrm{CE}\left(1,4 \mathrm{dS} \mathrm{m}^{-1}\right)$. Portanto, a diferença na massa seca provavelmente foi decorrente da maior disponibilidade de água do tratamento $0,4 \mathrm{~m}^{3} \mathrm{~m}^{-3}$, o que está de acordo com o trabalho de Moccaldi e Runkle (2009). Esses autores modelaram matematicamente o efeito da umidade do substrato, temperatura e radiação no crescimento de sálvia e tagetes, e encontraram que valores constantes ao longo do ciclo foram melhores para o crescimento do que flutuações dessas variáveis ao longo do tempo.

$\mathrm{O}$ tratamento acionado com maior umidade $\left(0,4 \mathrm{~m}^{3} \mathrm{~m}^{-3}\right)$ proporcionou aumento de $8 \%$ na altura da parte aérea das plantas $(\mathrm{p}<0,0001)$, o que era esperado e está de acordo com o trabalho de Alem et al. (2011) em plantas de petúnia. Não houve efeito significativo da altura da parte aérea medida na ponta da flor mais alta e na axila foliar mais alta $(\mathrm{p}>0,05$, Tabela 1). A área foliar total foi $18 \%$ significativamente superior no tratamento $0,4 \mathrm{~m}^{3} \mathrm{~m}^{-3}(\mathrm{p}=0,0007)$, o que já era esperado em razão da maior disponibilidade de água desse tratamento (Tabela 1). Esse resultado foi similar ao encontrado por Alem et al. (2011) para petúnia em um estudo com doses crescentes de fertilizante de liberação lenta e CVA do substrato. O número de ramos determinados para os dois tratamentos ao final do experimento não foi significativamente diferente entre os dois tratamentos $(\mathrm{p}>0,05)$. Esses resultados estão de acordo com Ferrarezi et al. (2013), que provaram que valores crescentes de CVA proporcionaram maior desenvolvimento vegetal na produção de porta-enxertos cítricos. Provavelmente o efeito negativo da variação de umidade e possível ocorrência de hipoxia na fotossíntese, também foi prejudicial para o desenvolvimento vegetal (PISICCHIO et al., 2010 e VAN IERSEL et al., 2010), resultando na ausência de diferenças entre os tratamentos para algumas variáveis. Dessa forma, indica-se o uso de outros sistemas de irrigação que permitam um melhor controle da umidade do substrato, como o gotejamento, por exemplo, que podem ser mais eficientes na realização de experimentos em câmara de crescimento para avaliação da fotossíntese em planta inteira.

Tabela 1. Massa seca das folhas (MSFOL), massa seca do caule + flores (MSC+F), massa seca total da parte aérea (MSTPA), altura da parte aérea na ponta da flor mais alta (APAPF), altura da parte aérea na axila foliar mais alta (APAX), área foliar total (AFT) e número de ramos (NR) de Salvia splendens Sell ex Roem. \& Schult. 'Vista Rose', determinadas no final do experimento. Média de 4 repetições contendo 10 plantas.

Table 1. Leaf dry weight (MSFOL), stem + flowers dry weight $(M S C+F)$, shoot total dry weight (MSTPA), shoot height at the tip of the tallest flower (APAPF), shoot height in the highest leaf armpit (APAX), total leaf area (AFT), and number of branches (NR), determined at the end of the experiment. Average of 4 replication and 10 plants.

\begin{tabular}{|c|c|c|c|c|c|c|c|}
\hline \multirow{2}{*}{ Tratamentos } & MSFOL & $\mathbf{M S C}+\mathbf{F}$ & MSTPA & APAPF & APAX & \multirow{2}{*}{$\begin{array}{c}\text { AFT } \\
\mathrm{cm}^{2}\end{array}$} & \multirow{2}{*}{ NR } \\
\hline & \multicolumn{3}{|c|}{------ g planta ${ }^{-1}$} & \multicolumn{2}{|c|}{------ cm ------- } & & \\
\hline $0,2 \mathrm{~m}^{3} \mathrm{~m}^{-3}$ & $1,62 \mathrm{NS}$ & $2,20 \mathrm{~b}$ & $3,82 \mathrm{~b}$ & $25,35 \mathrm{~b}$ & $13,67 \mathrm{NS}$ & $471,95 \mathrm{~b}$ & $5,37 \mathrm{NS}$ \\
\hline $0,4 \mathrm{~m}^{3} \mathrm{~m}^{-3}$ & $1,80 \mathrm{NS}$ & $2,44 \mathrm{a}$ & $4,24 \mathrm{a}$ & $27,30 \mathrm{a}$ & $14,10 \mathrm{NS}$ & $558,01 \mathrm{a}$ & $5,25 \mathrm{NS}$ \\
\hline$P>F$ & 0,0573 & 0,0123 & 0,0129 & $<0,0001$ & 0,0871 & 0,0007 & 0,7785 \\
\hline C.V. & $12,30 \%$ & $14,11 \%$ & $10,74 \%$ & $9,69 \%$ & $12,92 \%$ & $11,98 \%$ & $30,78 \%$ \\
\hline
\end{tabular}

Médias seguidas de mesma letra nas colunas indicam que os valores apresentados não diferiram entre si a 5\% de probabilidade. Onde: NS = não significativo.

\section{CONCLUSÕES}

Não houve diferença entre os tratamentos nos parâmetros fisiológicos avaliados. O tratamento acionado quando a umidade do substrato foi inferior a $0,4 \mathrm{~m}^{3} \mathrm{~m}^{-3}$ proporcionou maior massa seca, maior altura de plantas e maior área foliar total em comparação com o tratamento $0,2 \mathrm{~m}^{3} \mathrm{~m}^{-3}$. A subirrigação não se mostrou eficiente para imposição do estresse hídrico em câmaras de crescimento, pois ocasionou CVA acima do indicado para crescimento ideal em ambos os tratamentos. Experimentos futuros precisam ser conduzidos com outras espécies nas mesmas condições para avaliar esses efeitos sobre as plantas.

\section{AGRADECIMENTOS}

A Sue Dove, a Peter Otieno Alem e Karina Aparecida dos Santos Soranz pela colaboração técnica e a CAPES pela bolsa de doutorado-sanduíche e de pós-doutoramento do primeiro autor na The University of Georgia (Proc. BEX 1390/10-4 e BEX 2620/13-8). O financiamento da pesquisa foi realizado pela American Floral Endowment e USDANIFA-SCRI (Award No. 2009-51181-05768). 


\section{REFERÊNCIAS}

ALEM, P.O.; THOMAS, P.A.; VAN IERSEL, M.W. Growth of petunia as affected by substrate moisture content and fertilizer rate. HortScience, Alexandria, v.47, SCRI-6 (Abstr.), 2011.

ANDRIOLO, J.L.; LE BOT, J.; GARY, C.; SAPPE, G.; ORLANDO, P.; BRUNEL, B.; SARROUY, C. An experimental set-up to study carbon, water, and nitrate uptake rates by hydroponically grown plants. Journal of Plant Nutrition, Athens, v.19, p.1441-1462, 1996. http:// dx.doi.org/10.1080/01904169609365211>

CHAPPELL, M.; DOVE, S.K.; VAN IERSEL, M.W.; THOMAS, P.A.; RUTER, J. Implementation of wireless sensor networks for irrigation control in three container nurseries. HortTechnology, Alexandria, v.23, p.747-753, 2013.

CHAVES, M.M.; FLEXAS, J.; PINHEIRO, C. Photosynthesis under drought and salt stress: regulation mechanisms from whole plant to cell. Annals of Botany, London, v.103, p.551-560, 2009. http://dx.doi.org/10.1093/ $\mathrm{aob} / \mathrm{men} 125>$

FERRAREZI, R.S.; RIBEIRO, M.D.; VAN IERSEL, M.W.; TESTEZLAF, R. Subirrigation controlled by capacitance sensors for citrus rootstock production. HortScience, Alexandria, v.48, S142 (Abstr.), 2013.

FERRAREZI, R.S.; VAN IERSEL, M.W.; TESTEZLAF, R. Monitoring and controlling ebb-and-flow subirrigation with soil moisture sensors. HortScience, Alexandria, v.50, p.447-553, 2015.

FERRAREZI, R.S.; VAN IERSEL, M.W.; TESTEZLAF, R. Subirrigation automated by capacitance sensors for salvia production. Horticultura Brasileira, Brasília, v.32, p.314-320, 2014. http://dx.doi.org/10.1590/S0102$05362014000300013>$

GARCÍA-SÁNCHEZ, F.; SYVERTSEN, J.P.; GIMENO, V.; BOTÍA, P.; PEREZ-PEREZ, J.G. Responses to flooding and drought stress by two citrus rootstock seedlings with different water-use efficiency. Physiologia Plantarum, Malden, v.130, p.532-542, 2007. http://dx.doi.org/10.1111/ j.1399-3054.2007.00925.x>

KANG, J.G.; VAN IERSEL, M.W. Nutrient solution concentration affects shoot-root ratio, leaf area ratio, and growth of subirrigated salvia. HortScience, Alexandria, v.39, p.49-54, 2004.

KIM, J.; VAN IERSEL, M.W. Slowly developing drought stress increases photosynthetic acclimation of Catharanthus roseus. Physiologia Plantarum, Malden, v.143, p.166-177, 2011. http://dx.doi.org/10.1111/j.13993054.2011.01493.x
KIM, J.; VAN IERSEL, M.W.; BURNETT, S.E. Estimating daily water use of two petunia cultivars based on plant and environmental factors. HortScience, Alexandria, v.46, p.1287-1293, 2011.

LEA-COX, J.D.; BAUERLE, W.L.; VAN IERSEL, M.W.; KANTOR, G.F.; BAUERLE, T.L.; LICHTENBERG, E.; KING, D.M.; CRAWFORD, L. Advancing wireless sensor networks for irrigation management of ornamental crops: An overview. HortTechnology, Alexandria, v.23, p.717724, 2013.

MARQUES, P.A.A.; BORTOLO, D.P.G.; SANTOS, A.C.P. Produtividade de inflorescências de calêndula sob irrigação suplementar na região do oeste paulista. Irriga, Botucatu, v.16, p.153-162, 2011.

MOCCALDI, L.A.; RUNKLE, E.S. Modeling the effects of temperature and photosynthetic daily light integral on growth and flowering of Salvia splendens and Tagetes patula. Journal of the American Society for Horticultural Science, Alexandria, v.132, p.283-288, 2007.

NEMALI, K.S.; VAN IERSEL, M.W. Light effects on wax begonia: photosynthesis, growth respiration, maintenance respiration, and. carbon use efficiency. HortScience, Alexandria, v.129, n.416-424, 2004.

PISICCHIO, C.M.; BIANCHINI, E.; PIMENTA, J.A.; SERT, M.A.; DAVANSO-FABRO, V.M.; MEDRI, M.E. Heliocarpus popayanensis Kunth (Malvaceae) tolera a hipóxia do substrato? Acta Scientiarum Biological Sciences, Maringá, v.32, p.201-209, 2010. <http://dx.doi. org/10.4025/actascibiolsci.v32i2.3566>

SANTOS, R.F.; CARLESSO, R. Déficit hídrico e os processos morfológico e fisiológico das plantas. Revista Brasileira de Engenharia Agrícola e Ambiental, Campina Grande, v.2, p.287-294, 1998.

SNOW, M.D.; TINGEY, D.T. Evaluation of a system for the imposition of plant water stress. Plant Physiology, Waterbury, v.77, p.602-607, 1985.

VAN IERSEL, M.W.; BUGBEE, B. A multiple chamber, semicontinuous, crop $\mathrm{CO}_{2}$-exchange system: design, calibration, and data interpretation. Journal of the American Society for Horticultural Science, Alexandria, v.125, p.86-92, 2000.

VAN IERSEL, M.W.; DOVE, S.K.; KANG, J.G.; BURNETT, S.E. Growth and water use of petunia as affected by substrate water content and daily light integral. HortScience, Alexandria, v.45, p.277-282, 2010. 
VAN IERSEL, M.W.; KANG, J. Nutrient solution concentration affects whole-plant $\mathrm{CO}$ exchange and growth of subirrigated pansy. Journal of the American Society for Horticultural Science, Alexandria, v.127, p.423-429, 2002.
ZELITCH, I. The close relationship between net photosynthesis and crop yield. BioScience, Oxford, v.32, p.796-802, 1982. 